\title{
基于几何约束的蛋白质-配体准确结合自由能计算
}

\author{
付浩浩 $a$ 陈淏川 $a$ 张宏 ${ }^{a}$ 郡学广*, $a, b$ 蔡文生 ${ }^{*}, a$ \\ ( ${ }^{a}$ 南开大学化学学院分析科学研究中心 天津市生物传感与分子识别重点实验室 天津 300071) \\ ( $b$ 南开大学药物化学生物学国家重点实验室 天津 300071)
}

\begin{abstract}
摘要 蛋白质-配体的结合过程伴随着复杂的结构变化, 在分子模拟可及的时间尺度内难以完全捕获, 这使得准确估 计蛋白质-配体的结合自由能十分困难. 一种有效的解决途径是采用几何约束减小需要采样的构象空间, 再通过后处 理方式扣除约束的影响. 本文综述了三种几何约束策略——漏斗状约束、球形约束和七自由度约束与自由能计算算法 结合准确计算结合自由能的原理和进展, 重点概述理论严谨的七自由度约束的最新进展以及与 Alchemistry 或重要性 采样方法的联用策略, 最后, 讨论了如何针对不同体系选择合适的计算策略以及蛋白质-配体准确结合自由能计算在 药物设计等领域中的挑战和前景, 并提出了将上述方法进一步运用于研究更复杂的蛋白质-蛋白质问题的可能性.

关键词 蛋白质-配体; 结合自由能; 分子模拟; 自由能微扰; 重要性采样
\end{abstract}

\section{Accurate Estimation of Protein-ligand Binding Free Energies Based on Geometric Restraints}

\author{
Haohao $\mathrm{Fu}^{a}$ Haochuan $\mathrm{Chen}^{a} \quad$ Hong Zhang $^{a}$ Xueguang Shao*,a,b Wensheng Cai ${ }^{*, a}$ \\ ( ${ }^{a}$ Tianjin Key Laboratory of Biosensing and Molecular Recognition, Research Center for Analytical Sciences, \\ College of Chemistry, Nankai University, Tianjin 300071, China) \\ ( ${ }^{b}$ State Key Laboratory of Medicinal Chemical Biology, Tianjin 300071, China)
}

\begin{abstract}
Binding free energy is the most crucial physical quantity for describing recognition-association of protein-ligand hybrids. Accurate estimation of protein-ligand binding free energies is of paramount importance in the field of drug design and biological engineering. However, the association process of protein-ligand hybrids is usually coupled with complex conformational changes of molecular objects, which is not amenable to the timescale of classical molecular simulations. This limitation makes it difficult to accurately estimate the protein-ligand binding free energies using classical free-energy calculation strategies. An effective solution is to apply geometric restraints to reduce the configurational space needed to be sampled, so as to boost up the convergence rate of simulations, and then calculate and deduct the contribution of these restraints to the binding free energy by post-processing. In this review, we firstly introduce the recent developments of three geometric restraints, namely, funnel, spherical, and seven-degree-of-freedom restraints, used in accurate binding free-energy calculations, with emphasis on the latest progress of the third one. Specifically, the theoretically rigorous seven-degree-of-freedom restraint describes translational, orientational, rotational, and conformational degrees of freedom by means of a center-of-mass distance, spherical angles, Euler angles and the root-mean-square deviation. Moreover, we demonstrate the theoretical backgrounds and methods of how to achieve accurate protein-ligand binding free-energy estimation by combination of geometric restraints and importance-sampling or alchemical algorithms. In the geometric routes, the degrees of freedom of the relative movement of the protein-ligand complex are addressed in a stepwise fashion by one-dimensional importance-sampling simulations. In the alchemical routes, a special thermodynamics cycle is designed, in which additional simulations are performed to address the contribution of the restraints. A general suggestion for how to choose a suitable strategy for a given molecular assembly based on our experience is provided. Last but not least, we discuss the applications and challenges of using accurate protein-ligand binding free-energy calculation methods in fields such as drug design, and present the possibility of extending these methods for investigating complex protein-protein interaction.
\end{abstract}

Keywords protein-ligand; binding free energy; molecular dynamics; free-energy perturbation; importance sampling

\section{1 引言}

结合自由能(binding free energy)是描述识别-组装
过程最重要的物理量, 理论估计结合自由能在许多领域 都至关重要 ${ }^{[1-4]}$. 例如在药物设计过程中, 常通过分子对 接或分子模拟计算数据库中的备选药物与受体蛋白的

\footnotetext{
*E-mail: xshao@nankai.edu.cn, wscai@nankai.edu.cn, Tel.: 022-23503430

Received October 24, 2020; published November 30, 2020.

Project supported by the National Natural Science Foundation of China (Nos. 22073050, 21773125), the Fundamental Research Funds for the Central Universities, Nankai University (Nos. 63191743, 63201015), and the China Post-doctoral Science Foundation (No. bs6619012).

项目受国家自然科学基金(Nos. 22073050, 21773125)、南开大学中央高校基本科研业务费专项资金(Nos. 63191743, 63201015)和中国博士后科学基金 (No. bs6619012)资助.
} 
结合自由能, 并以此作为判据篎选出可能的药物 ${ }^{[5]}$. 在 蛋白质工程领域, 可通过对蛋白质关键氨基酸进行突 变, 并进行结合自由能计算, 观测结合能力的变化, 从 而指导对蛋白质的改性设计 ${ }^{[6]}$. 显而易见, 蛋白质-配体 结合自由能计算的准确性直接影响其在各领域中的应 用前景. “准确” 一词有两个方面的含义: 一是经典力 场或打分函数可以准确地描述微观粒子的相互作用, 二 是模拟过程完整地捕获了蛋白质-配体结合过程各个自 由度的贡献, 并准确地描述统计力学行为. 其中前者属 于力场拟合范畴的问题, 近年来发展的极化力场对非键 相互作用有着较好的描述 ${ }^{[7-8]}$, 该问题在参考文献 $[7,9]$ 中已有了详尽的阐述, 本综述仅聚焦于后者在近年来的 发展与未来的展望.

从统计力学的角度看, 蛋白质-配体的标准结合自 由能可以通过下式计算:

$$
\Delta G_{\text {binding }}^{\circ}=-k_{\mathrm{B}} T \ln K^{\circ}=-k_{\mathrm{B}} T \ln \frac{p_{\text {bound }}}{p_{\text {unbound }}}
$$

其中 $\Delta G_{\text {binding }}^{\circ}$ 为标准结合自由能, $k_{\mathrm{B}}$ 为玻尔兹曼常数, $T$ 为温度, $K^{\circ}$ 为标准平衡常数, $p_{\text {bound }}$ 和 $p_{\text {unbound 分别为 }}$ 平衡状态下结合态和非结合态出现的概率. 然而, 直接 通过式(1)计算结合自由能在实际中十分困难, 因为在 可及的分子模拟时间尺度内, 很难看到结合态和非结合 态间的自发转化 ${ }^{[1]}$. 应用重要性采样方法(importance sampling $)^{[10]}$, 如 metadynamics ${ }^{[11-12]}$ 或自适应偏置力 (adaptive biasing force, ABF) $)^{[13-14]}$, 可以加速蛋白质-配 体的结合-分离过程, 实现在分子模拟的时间尺度内观 察到该过程. 然而由于在结合-分离过程中, 蛋白质-配 体间还伴随着复杂的取向和构象变化, 而在现有的计算 条件下, 重要性采样模拟不可能同时捕获沿平动、转动 和构象变化自由度方向上的运动, 使得直接使用重要性 采样方法计算蛋白质-配体的结合自由能亦十分困难 ${ }^{[15]}$.

另一种标准结合自由能计算的思路为使用 Alchemistry 类方法, 如自由能微扰(free-energy perturbation, FEP) ${ }^{[16]}$ 和热力学积分(thermodynamics integration, TI $)^{[17]}$, 通过在体系中 “消失” 或 “生成”一部分原 子, 收集模拟过程中体系的内能变化, 进一步计算 “消 失” 或 “生长” 过程对应的自由能变化. 在实际应用中, 常通过设计热力学循环, 进行多次 alchemistry 计算, 得 到目标结合自由能, 如图 1 所示, 可以通过计算 $\Delta G_{1}$ 和

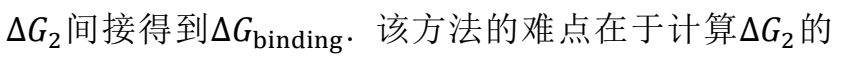

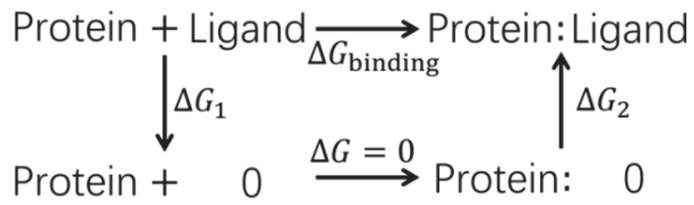

图 1 用 Alchemistry 方法计算蛋白质-配体结合自由能的传统策略. “:”表示结合态

Figure 1 The classical strategy of protein-ligand binding free-energy calculations using alchemical algorithms. "“" represents the binding state
过程中，随着配体的逐渐 “消失”，配体相对于蛋白质 会出现复杂的平动、转动和构象变化等过程. 而在分子 模拟的时间尺度内难以捕获这些过程，从而阻碍 Alchemistry 类方法计算的收敛性.

为解决重要性采样或 Alchemistry 算法在计算蛋白 质-配体结合自由能过程中的收敛性难题，可以采用几 何约束减少需要采样的构象空间, 并通过后处理准确地 扣除约束对计算结果的影响. 近年来陆续提出了漏斗状 (funnel)约束 ${ }^{[18-19]}$, 球状(spherical)约束 ${ }^{[20]}$, 六/七自由度 (six/seven degree of freedom)约束 ${ }^{[21-23]}$ 等. 这些约束分别

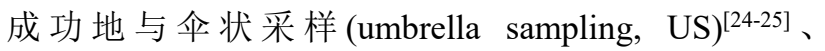
metadynamics ${ }^{[11-12]}$ 、自适应偏置力 ${ }^{[13-14]}$ 、自由能微扰 ${ }^{[16] 、}$ 热力学积分 ${ }^{[17]}$ 等重要性采样或 alchemistry 算法联用, 准 确计算了大量蛋白质-配体的结合自由能 ${ }^{[26-30]}$.

本综述首先从原理、特点、实现、应用范围和发展 上对近年来提出的几何约束方式和后处理策略, 以及将 这些约束与重要性采样或 alchemistry 算法联用的方法 进行了综述，然后重点阐述了理论严谨的七自由度约束 与自适应偏置力和自由能微扰算法联用的策略, 最后对 准确进行蛋白质-配体结合自由能计算的方法在未来的 发展方向进行了总结和展望.

\section{2 漏斗状约束和球形约束与重要性采样方法联 用计算结合自由能}

\section{1 漏斗状约束与 funnel-metadynamics}

在重要性采样模拟过程中, 如果选用蛋白质-配体 的质心距离作为集合变量(collective variables, 或 order parameters), 在配体远离蛋白质的过程中, 配体会相对 于蛋白质发生公转, 导致当配体再次靠近蛋白质时, 无 法寻找到正确的结合位点. 为解决该问题, Parrinello 课 题组提出了漏斗状约束 ${ }^{[18-19]}$ (图 2A), 该约束避免了配体 的公转, 保证配体在靠近蛋白质的过程中能够正确地寻 找到结合位点, 从而使得重要性采样算法可以可逆地探 索蛋白质-配体的结合-解离过程. 漏斗状约束的贡献可
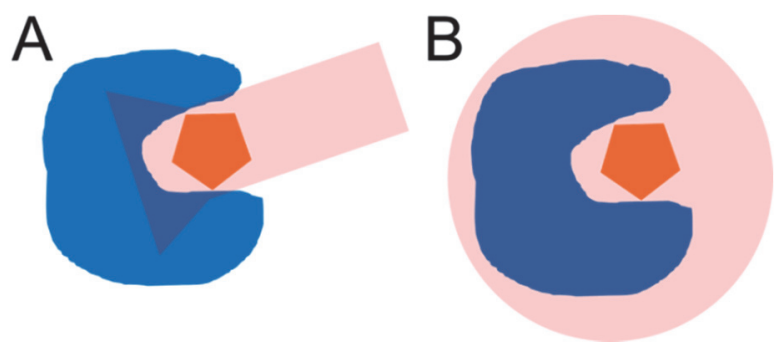

图 2 (A)漏斗状约束和(B)球形约束示意图. 浅红色区域为允许小分 子自由运动的区域. 若小分子质心位于浅红色区域外, 则会受到指向 浅红色区域的边界约束力

Figure 2 (A) Funnel and (B) spherical restraints used in binding free-energy calculations. Region for free move of the ligand is shown in red. The ligand will be pushed back by the restraint if it moves outside the red region. 
以通过式(2)计算

$$
\Delta G_{\text {restraint }}=k_{\mathrm{B}} T \ln \left(\pi R^{2} C^{\circ}\right)
$$

其中 $\Delta G_{\text {restraint }}$ 为约束对结合自由能的贡献, $R$ 为圆柱体 半径, $C^{\circ}=1000 / 1661 \mathrm{~nm}^{-3}$ 为标准浓度.

原理上来说, 漏斗状约束可以与任意重要性采样方 法联用, 但由于该约束与 metadynamics 为同一课题组所 提出, 所以常与 metadynamics 联用, 称为 funnel-metadynamics ${ }^{[18-19]}$. Funnel-metadynamics 计算中 需要纳入配体质心与结合位点的距离作为集合变量之 一, 此外也可以根据需要加入球面角、配体骨架二面角 等其他重要自由度, 通过 metadynamics 计算得到一维或 多维自由能曲面. 在一维情况下, 通过下式计算结合自 由能:

$$
\Delta G_{\text {binding }}=-k_{\mathrm{B}} T \ln \left[\frac{\int_{\text {bound }} \mathrm{d} \xi \mathrm{e}^{\frac{-w(\xi)}{k_{\mathrm{B}} T}}}{\int_{\text {unbound }} \mathrm{d} \xi \mathrm{e}^{\frac{-w(\xi)}{k_{\mathrm{B}} T}}}\right]
$$

其中 $\xi$ 为使用的集合变量, 即距离, $w(\xi)$ 为自由能曲线. 需要注意的是由式(3)计算得到的结合自由能需要扣除 式(2)中的约束项贡献并利用式(4)进行标准态校正.

$$
\begin{gathered}
\Delta G_{\text {binding }}=-k_{\mathrm{B}} T \ln K_{\text {eq }} \\
\Delta G_{\text {binding }}^{\circ}=-k_{\mathrm{B}} T \ln K_{\text {eq }}^{\circ}=-k_{\mathrm{B}} T \ln \left(K_{\text {eq }} C^{\circ}\right)
\end{gathered}
$$

其中 $K_{\mathrm{eq}}{ }^{\circ}$ 为标准平衡常数.

Funnel-metadynamics 的优点为几何约束意义明确、 使用简单、消耗计算资源少, 对蛋白质-小分子配体的结 合自由能计算有着较好的效果. 在 Plumed 自由能计算 模块 ${ }^{[31]}$ 中有 funnel-metadynamics 的直接实现, 由于几何 约束形式简单, 在 Colvars $^{[32]}$ 、SSAGES ${ }^{[33]}$ 等自由能计算 模块中也有较为简便的方式直接使用. 因此, Funnelmetadynamics 可以无障碍地被大多数现有的分子模拟 软件所支持. 为进一步简化 funnel-metadynamics 的使 用, 促进准确结合自由能计算在更多领域的应用, Raniolo 等人为设计了简化 funnel-metadynamics 输入文件步 骤的软件, 并在 Nature Protocols 上发表了准确结合自由 能计算的详细教程 ${ }^{[19]}$. 目前 funnel-metadynamics 已发展 成为研究膜蛋白, 特别是 $G$ 蛋白偶联受体 (G Protein-Coupled Receptors, GPCRs)与配体相互作用最为常 见的方法 ${ }^{[34]}$.

Funnel-metadynamics 的主要缺点为没有针对配体 的取向和构象变化进行描述. 如果配体分子较大或者柔 性较强, 其取向和构象变化会阻碍 metadynamics 模拟过 程中对最优结合模式的采样, 从而影响自由能计算的收 玫. 此外, 如果配体存在不止一个结合位点的话, funnel-metadynamics 也很难对配体在其他结合位点上的结 构进行采样.

\section{2 球形约束与重要性采样方法结合}

为解决配体存在多个结合位点的问题, Parrinello 课

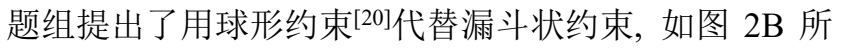

示. 球形约束对结合自由能的贡献如式(5)所示.

$$
\Delta G_{\text {restraint }}=k_{\mathrm{B}} T \ln \left[C^{\circ}\left(\frac{4}{3} \pi R^{3}-V_{\text {protein }}\right)\right]
$$

其中 $V_{\text {protein }}$ 为蛋白质所占球体内部的体积.

通过结合球形约束和重要性采样算法, 如 metadynamics 和自适应偏置力, 使用球面坐标 $(r, \theta, \varphi)$ 作为集合变量进行多维自由能计算可以寻找到配体与 蛋白质的多个结合位点, 通过对三维自由能曲面积分, 可以得到准确的结合自由能, 如下式所示,

$$
\Delta G_{\text {binding }}=-k_{\mathrm{B}} T \ln \left[\frac{\iint_{\text {bound }} \mathrm{d} r \mathrm{~d} \theta \mathrm{d} \varphi \mathrm{e}^{\frac{-w(r, \theta, \varphi)}{k_{\mathrm{B}} T}}}{\iint_{\int_{\text {unbound }} \mathrm{d} r \mathrm{~d} \theta \mathrm{d} \varphi \mathrm{e}^{\frac{-w(r, \theta, \varphi)}{k_{\mathrm{B}} T}}}}\right]
$$

经过式(4)和式(5)的校正即可得到标准结合自由能.

球形约束解决了漏斗状约束难以对多个结合位点 进行采样的问题, 并且几何约束形式更加简单, 在几乎 所有的分子动力学软件中均得到支持. 但是球形约束依 然没有解决复杂配体在结合-解离过程中可能存在取向 和构象变化的问题, 因此对于复杂或柔性较大的配体, 自由能计算依旧会存在收敛困难，导致计算结果不够准 确.

\section{3 六/七自由度约束及在自由能计算中的应用}

\section{1 六/七自由度约束}

如果将蛋白质和配体均视为刚体的话, 则采用球面 坐标 $(r, \theta, \varphi)$ 可以完备地描述蛋白质和配体的相对位置, 采用配体相对于蛋白质的欧拉角 $(\Theta, \Phi, \Psi)$ 可以完备地 描述配体的相对取向. 因此, 通过向这六个自由度施加 合理的约束, 可以大大减少模拟中所需要采样的构象空 间，从而加速模拟的收敛.

使用六自由度约束的首要问题是如何定义这六个 自由度. Karplus 课题组提出可以在蛋白质和配体中分别 选择 3 个原子团作为参考点, 利用这 6 个参考点定义 2 个球面角和 3 个欧拉角 ${ }^{[21]}$, 如图 3 所示. 这种方法假定 蛋白质和配体内的 3 个原子团自身的相对位置不变, 即 蛋白质和配体均为刚体, 在配体柔性较大的情况下该条 件很难满足. 为此, Roux 课题组进一步将配体结构相对 于结合态的均方根偏差 (root-mean-square deviation, RMSD)变化也加入约束中 ${ }^{[22,35]}$, 即七自由度约束. 采用 参考原子团定义蛋白质-配体相对运动的自由度实现简 单, 可以直接被各主流分子动力学软件所支持. 由于物 理意义明确, 理论严谨, 六/七自由度约束已逐渐发展成 为计算蛋白质-配体结合自由能事实上的标准约束方 式[36,37].

\section{2 七自由度约束与重要性采样方法相结合准确计算 结合自由能}

Chipot 课题组通过将蛋白质-配体的相对运动过程 中涉及到的七个自由度分解, 通过分步策略 ${ }^{[38]}$, 采用重 


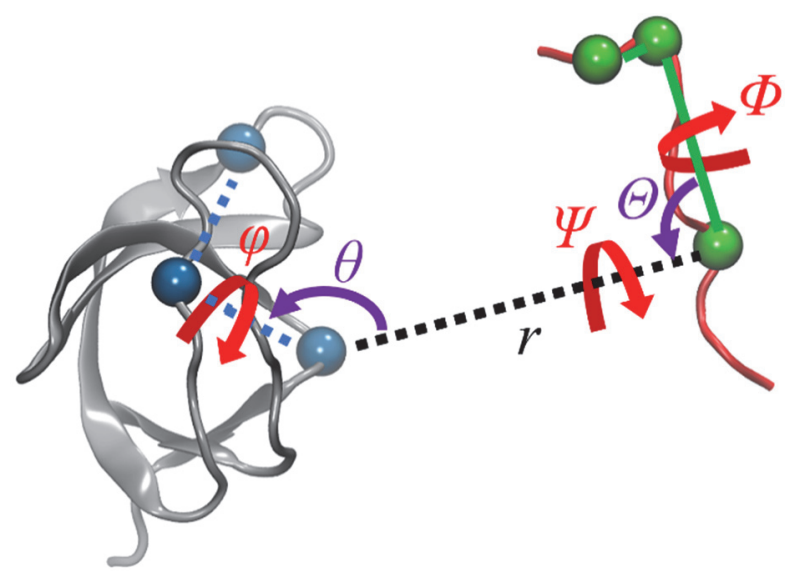

图 3 基于参考原子团定义的六自由度 $(r, \theta, \varphi, \Theta, \Phi, \Psi)$ 示意图. 图中 黑色虚线表示距离, 紫色箭头表示角度, 红色箭头表示二面角

Figure 3 Atom-group-based definition of the six degrees of freedom $(r, \theta, \varphi, \Theta, \Phi, \Psi)$. The black dot line, red arrows and purple arrows represent the distance, angles and dihedral angles, respectively.

要性采样算法分别沿每个自由度进行采样. 原理如式 (7)所示, 蛋白质-配体的标准结合常数可分解为多个项 相乘:

$$
\begin{aligned}
& K_{\mathrm{eq}}=\frac{\int_{\text {site }} d \mathbf{1} \int d \mathbf{x ~}^{-\beta U}}{\int_{\text {site }} d \mathbf{1} \delta\left(\mathbf{x}_{1}-\mathbf{x}_{1}^{*}\right) \int d \mathbf{x} e^{-\beta U}} \\
& =\frac{\int_{\text {site }} d \mathbf{1} \int d \mathrm{xe}^{-\beta U}}{\int_{\text {site }} d \mathbf{1} \int d \mathrm{x}^{-\beta\left(U+u_{c}\right)}} \\
& \times \frac{\int_{\text {site }} d \mathbf{1} \int d \mathrm{xe}^{-\beta\left(U+u_{c}\right)}}{\int_{\text {site }} d \mathbf{1} \int d \mathrm{x}^{-\beta\left(U+u_{c}+u_{o}\right)}} \\
& \times \frac{\int_{\text {site }} d \mathbf{1} \int d \mathbf{x}^{-\beta\left(U+u_{c}+u_{o}\right)}}{\int_{\text {site }} d \mathbf{1} \int d \mathbf{x e}^{-\beta\left(U+u_{c}+u_{o}+u_{a}\right)}} \\
& \times \frac{\int_{\text {site }} d \mathbf{1} \int d \mathbf{x}^{-\beta\left(U+u_{c}+u_{o}+u_{a}\right)}}{\int_{\text {bulk }} d \mathbf{1} \delta\left(\mathbf{x}_{1}-\mathbf{x}_{1}^{*}\right) \int d \mathbf{x ~}^{-\beta\left(U+u_{c}+u_{o}\right)}} \\
& \times \frac{\int_{\text {bulk }} d \mathbf{1} \delta\left(\mathbf{x}_{1}-\mathbf{x}_{1}^{*}\right) \int d \mathbf{x e}^{-\beta\left(U+u_{c}+u_{o}\right)}}{\int_{\text {bulk }} d \mathbf{1} \delta\left(\mathbf{x}_{1}-\mathbf{x}_{1}^{*}\right) \int d \mathbf{x ~}^{-\beta\left(U+u_{c}\right)}} \\
& \times \frac{\int_{\text {bulk }} d \mathbf{1} \delta\left(\mathrm{x}_{1}-\mathrm{x}_{1}^{*}\right) \int d \mathbf{x}^{-\beta\left(U+u_{c}\right)}}{\int_{\text {bulk }} d \mathbf{1} \delta\left(\mathrm{x}_{1}-\mathrm{x}_{1}^{*}\right) \int d \mathbf{x ~}^{-\beta(U)}} \\
& =\exp \left[-\beta\left(\begin{array}{c}
\Delta G_{c}^{\text {site }}+\Delta G_{o}^{\text {site }}+\Delta G_{a}^{\text {site }} \\
-\frac{1}{\beta} \ln \left(S^{*} I^{*} C^{\circ}\right) \\
+\Delta G_{o}^{\text {bulk }}+\Delta G_{c}^{\text {bulk }}
\end{array}\right)\right]
\end{aligned}
$$

其中 $\mathbf{1}$ 代表配体, $\mathbf{x}_{1}$ 为配体的质心, $\mathbf{x}_{1}^{*}$ 为体系中远离结 合位点的一个任意点, $U$ 为势能, $u_{o}=u_{\Theta}+u_{\Phi}+u_{\Psi}$,
是配体的取向约束, $u_{a}=u_{\theta}+u_{\varphi}$, 为相对位置约束, $\Delta G_{c} 、 \Delta G_{o}$ 和 $\Delta G_{a}$ 分别为构象、取向和相对位置约束的贡 献. 在实际计算中, 通过分步策略, 每一次模拟沿描述 一个自由度的集合变量 $\xi$ 进行重要性采样计算，而已经 计算过的自由度则约束在最优位置, 详细的计算步骤如 表 1 所示.

表 1 采用七自由度约束结合重要性采样算法准确计算蛋白质-配体 标准结合自由能的步骤

Table 1 Detailed process of the accurate protein-ligand binding free-energy calculation strategy using the combination of the sevendegree-of-freedom restraints and an importance-sampling algorithm

\begin{tabular}{ccccc}
\hline 步骤 & 对应项 & 集合变量 & 约束 & 体系 \\
\hline 1 & $\Delta G_{c}^{\text {site }}$ & RMSD & - & 蛋白质-配体 \\
2 & $\Delta G_{\Theta}^{\text {site }}$ & $\Theta$ & RMSD & 蛋白质-配体 \\
3 & $\Delta G_{\Phi}^{\text {site }}$ & $\Phi$ & RMSD, $\Theta$ & 蛋白质-配体 \\
4 & $\Delta G_{\psi}^{\text {site }}$ & $\Psi$ & RMSD, $\Theta, \Phi$ & 蛋白质-配体 \\
5 & $\Delta G_{\theta}^{\text {site }}$ & $\theta$ & RMSD, $\Theta, \Phi$, & 蛋白质-配体 \\
& & & $\Psi$ & \\
6 & $\Delta G_{\varphi}^{\text {site }}$ & $\varphi$ & RMSD, $\Theta, \Phi$, & 蛋白质-配体 \\
7 & $-\frac{1}{\beta} \ln \left(S^{*} I^{*} C^{\circ}\right)$ & $r$ & $\mathrm{RMSD}, \Theta, \Phi$, & 蛋白质-配体 \\
& & & $\Psi, \theta, \varphi$ & 配体 \\
8 & $\Delta G_{c}^{\text {bulk }}$ & $\mathrm{RMSD}$ & - & 解析计算 \\
\hline & $\Delta G_{o}^{\text {bulk }}$ & - & - &
\end{tabular}

相比于漏斗约束和球形约束, 采用七自由度策略结 合重要性采样算法进行结合自由能计算对蛋白质-配体 间的相对运动、相对取向变化和构象变化均进行了充分 描述. 而且在每个计算步骤中只用显式捕获一个自由度 的采样，之前计算过的其他自由度将被约束在最优的位 置, 从而避免了同时考虑多个自由度的多维自由能计 算, 保证了所需采样空间较小、采样充分、结果精确. 这 些约束的贡献(即各个自由度空间的贡献)通过后处理进 行综合、严谨的扣除. 因此七自由度策略结合重要性采 样收敛速度快，计算资源消耗少，适用于复杂体系.需 要注意的是如果复合物的结合-解离过程存在七自由度 以外的缓慢自由度时, 应添加额外的计算以捕获体系在 该自由度上的运动. 七自由度策略中仅用 RMSD, 角度 和距离等简单几何量定义蛋白质-配体间的自由度, 在 常用的自由能计算模块, 即 Plumed ${ }^{[31]}$, Colvars ${ }^{[32]}$ 和 SSAGES ${ }^{[33]}$ 中均有实现，因此被几乎所有的主流分子动 力学引擎所支持.

七自由度约束, 以及上文提到的漏斗状约束和球形 约束结合重要性采样算法在结合自由能计算中最主要 的缺点为这些算法仅适用于配体结合在蛋白表面的复 合物，而对于配体深埋在蛋白质内部的复合物，由于结 合-解离的过程过于复杂, 用重要性采样算法难以直接 进行模拟. 此外, 七自由度约束策略结合重要性采样算 法的另一个缺点为需要人为定义欧拉角和球面角, 这要 求对蛋白质的结构有一定的预先了解, 使用起来较为复 杂，且分步策略执行繁琐，对于非专业人士的可操作性 较差. 


\section{3 六/七自由度约束与 alchemistry 方法联用计算结 合自由能}

重要性采样算法不适用于结合位点在蛋白质内部 的蛋白质-配体结合自由能计算. 由于 alchemistry 方法 直接 “消失” 或 “生成” 原子, 无需经历有物理意义的 路径, 所以对于这种情况, 可以采用 alchemistry 方法进 行计算. Karplus 课题组提出了采用六自由度结合 alchemistry 方法计算蛋白质-配体标准结合自由能的策 略, 所采用的热力学循环如图 4 所示 ${ }^{[39]}$.

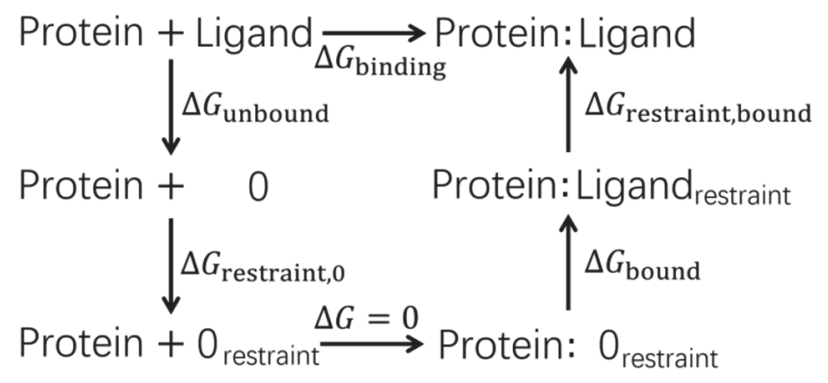

图 4 六/七自由度约束与 alchemistry 方法联合计算蛋白质-配体结合 自由能的热力学循环图

Figure 4 Thermodynamic cycle of the protein-ligand binding free-energy calculation using the combination of the six-/seven-degree-of-free restraints and an alchemical algorithm

图 4 中 $\Delta G_{\text {bound }}$ 和 $\Delta G_{\text {unbound }}$ 直接由 alchemistry 方法 计算得到, $\Delta G_{\text {restraint }, 0}$ 可以通过下式计算得到:

$$
\Delta G_{\text {restraint }, 0}=-k_{\mathrm{B}} T \ln \left[\frac{8 \pi^{2} V \sqrt{\left(K_{r} K_{\theta} K_{\varphi} K_{\Theta} K_{\Phi} K_{\Psi}\right)}}{r_{0}^{2} \sin \theta_{0} \sin \varphi_{0} \times\left(2 \pi k_{B} T\right)^{3}}\right]
$$

其中 $K$ 为约束力常数, 下标 0 代表初始位置.

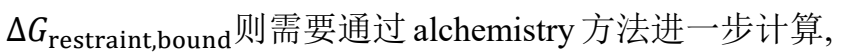
即通过逐渐地 “消失” 或 “生长” 约束, 计算两个态之 间的自由能变化. 该策略在计算刚性小分子配体的结合 自由能时有非常好的效果. 由于没有纳入 RMSD 自由 度, 这使得该方法在面对柔性较大的配体时存在收玫问 题.

Gumbart 等[38]针对该问题提出了一个完整的框架予 以解决, 即按照表 4 的步骤, 通过 alchemistry 方法计算 $\Delta G_{\text {bound }} 、 \Delta G_{\text {unbound }} 、 \Delta G_{\text {restraint }, 0}$ 和 $\Delta G_{\text {restraint,bound }}$. 在 该策略中，可以同时向体系中 “消失” 或 “生长” 全部 的七个自由度, 即 (RMSD, $r, \theta, \varphi, \Theta, \Phi, \Psi)$, 从而极大程 度上减少 alchemistry 计算过程中需要采样的构象空间, 加快收玫速度, 同时约束的影响又全部通过 alchemistry 方法严谨地计算得到. 该方法的步骤如表 2 所示.

与重要性采样相比, alchemistry 算法收玫较慢, 因 此在同等条件下, 选择重要性采样算法一般会得到略微 更好的结果 ${ }^{[38]}$. Deng 等 ${ }^{[27]}$ 对两种策略的对比也印证了 这一点. 然而, 由于使用重要性采样算法需要配体的
表 2 采用七自由度约束结合 alchemistry 算法准确计算蛋白质-配体 标准结合自由能的步骤

Table 2 Detailed process of the accurate protein-ligand binding free-energy calculation strategy using the combination of the sevendegree-of-freedom restraints and an alchemical algorithm

\begin{tabular}{|c|c|c|c|}
\hline 步骤 & 对应项 & 消失/生长部分 & 体系 \\
\hline 1 & $\Delta G_{\text {bound }}$ & 配体 & 蛋白质-配体 \\
\hline 2 & $\Delta G_{\text {unbound }}$ & 配体 & 配体 \\
\hline 3 & $\Delta G_{\text {restraint,bound }}$ & 七自由度约束 & 蛋白质-配体 \\
\hline 4 & $\begin{array}{c}\Delta G_{\text {restraint, } 0}(\mathrm{RMSD} \\
\text { 贡献) }\end{array}$ & RMSD 约束 & 配体 \\
\hline 5 & $\begin{array}{l}\Delta G_{\text {restraint, } 0}(\mathrm{RMSD} \\
\text { 贡献以外部分) }\end{array}$ & 无 & 解析计算(式 8) \\
\hline
\end{tabular}

结合位点在蛋白质表面，限制较大，基于约束的 alchemistry 方法和图 4 中的热力学循环已逐渐成为计算 蛋白质-配体结合自由能的标准策略 ${ }^{[36]}$. 基于该策略的 一个重要发展方向为准确结合自由能计算的 “流程化” 和 “工业化”，从而促进准确结合自由能计算在药物设 计领域的广泛应用. 为此, Sherman 和 York 课题组在 Amber 20 软件中面向药物设计的需求实现了结合自由 能计算的标准流程 ${ }^{[37]}$. 由 Chodera 课题组开发的 YANK 模块在简化操作流程的同时，结合了大量前沿的增强采 样方法, 为工业上所需的高通量篮选提供了可能性 ${ }^{[40]}$.

\section{4 约束-拉开-释放策略}

除表 1 和表 2 中介绍的基于重要性采样和 alchemistry 算法的策略以外, Dill 课题组和 Gilson 课题 组分别提出了约束-释放 (confine-and-release) ${ }^{[41]}$ 和约束拉开-释放(attach-pull-release) ${ }^{[42-43]}$ 策略. 在约束-拉开-释 放策略当中, 蛋白质和配体的解离过程被分解为 3 个可 逆步骤:

(a) 用 alchemistry 算法向配体施加 (RMSD, $\theta, \varphi, \Theta, \Phi, \Psi)$ 约束，方法同表 2 中步骤(3);

(b) 用重要性采样算法, 以 $r$ 为集合变量分离蛋白 质-配体复合物, 方法同表 1 中步骤(7);

(c) 用 alchemistry 算法释放配体的约束, 方法同表 2 中步骤(4)和(5).

可见约束-拉开-释放策略的本质为结合基于重要性 采样算法和基于 alchemistry 的策略. 与只使用 alchemistry 的策略 (表 2) 相比, 将计算 $\Delta G_{\text {bound }}$ 和

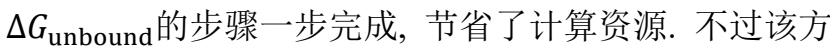
法无法应用于配体结合位点位于蛋白质内部的体系. 针 对该问题, Cruz 等人提出首先确定蛋白质中阻碍直线分 离蛋白质-配体复合物的原子团, 即 “障碍物” 基团. 在 步骤 $\mathrm{b}$, 即分离蛋白质-配体之前, 采用 alchemistry 方法 “消失” 掉障碍物基团, 从而保证分离步骤的顺利进行, 并计算该过程的自由能变化. 在步骤 $\mathrm{b}$ 之后采用 alchemistry 方法重新 “生长” 回这些基团 ${ }^{[44]}$. 不过该解 决方案势必会使得步骤更加复杂化, 与 3.2 和 3.3 节中阐 述的步骤相比是否还有优势函待考究. 


\section{5 基于质心向量和最优旋转的七自由度约束}

采用七自由度方法结合重要性采样或者 alchemistry 方法是一种理论严谨且实际高效的蛋白质-配体结合自 由能计算策略. 然而, 七自由度约束依赖在蛋白质和配 体中选择的参考基团, 需要用户对蛋白质和配体的结构 有提前的了解, 且参考基团的选择有可能对结果产生影 响. 另外使用欧拉角表示配体的自转, 在某些情况下会 发生“万向锁”现象, 导致欧拉角失效. 为解决该问题, 本课题组提出了基于质心向量和最优旋转的七自由度 约束 ${ }^{[23]}$, 并将其应用到蛋白质-配体结合自由能计算当 中.

蛋白质和配体的相对位置, 即球面角, 可以由连接 蛋白质-配体质心的单位向量 $\mathbf{d}_{\mathrm{unit}}=(x, y, z)$ 计算得到:

$$
\left\{\begin{array}{c}
\theta=\arccos (z) \\
\varphi=\operatorname{atan} 2(y, x)
\end{array}\right.
$$

为描述配体的相对旋转, 即欧拉角, 首先需计算由 当前状态到初始状态的最优旋转四元数 $\left(q_{0}, q_{1}, q_{2}, q_{3}\right)$, 这一步骤与计算 RMSD 过程中求解最优旋转步骤相 同 ${ }^{[45]}$. 欧拉角可以通过四元数计算而得:

$$
\left\{\begin{array}{c}
\Theta=\arcsin \left[2\left(q_{0} q_{2}-q_{3} q_{1}\right)\right] \\
\Phi=\operatorname{atan} 2\left[2\left(q_{0} q_{1}+q_{2} q_{3}\right), 1-2\left(q_{1}^{2}+q_{2}^{2}\right)\right] \\
\Psi=\operatorname{atan} 2\left[2\left(q_{0} q_{3}+q_{1} q_{2}\right), 1-2\left(q_{2}^{2}+q_{3}^{2}\right)\right]
\end{array}\right.
$$

式(9)中所定义的质心向量由蛋白质和配体的质心 计算而得, 式(10)中所定义的欧拉角由拟合所有配体原 子的最优旋转而得. 因此, 使用由式(9)和式(10)所定义 的变量, 仅需指定属于蛋白质和配体的原子, 无需额外 定义参考原子团, 使得结合自由能计算更加便捷, 且具 有更好的可重复性. 更重要的是, 由式(10)所定义的欧 拉角在结合态时, $(\Theta, \Phi, \Psi)=(0,0,0)$, 由于发生万向 锁时 $\Theta= \pm 90^{\circ}$, 所以采用式(10)的定义可以最大限度的 避免万向锁事件的发生.

采用上述约束同样可以利用表 1 和表 2 中所示的策 略与重要性采样算法或 alchemistry 算法结合, 从而实现 标准结合自由能计算, 相关算法已在 Colvars 自由能计 算模块中实现(目前被 Gromacs、Lammps 和 NAMD 所 支持). 由于使用式(9)和式(10)仅需用户分别指定属于 蛋白质和配体的原子, 因此采用式(9)和式(10)描述的约 束, 表 1 和表 2 所示的策略的过程可以实现自动化. 基 于此, 本课题组开发了 Binding free energy estimator(BFEE)结合自由能计算插件 ${ }^{[46]}$, 该插件仅需用户给 出蛋白质-配体复合物的结构, 即可自动生成计算所需 的所有输入文件, 待用户进行计算后, 该插件可以自动 进行后处理, 直接计算出结合自由能, 从而避免了使用 六/七自由度约束过程繁琐的操作步骤.

\section{4 无需约束的近似结合自由能计算方法}

进行蛋白质-配体的准确结合自由能计算复杂且昂
贵. 在许多情况下并不需要得到十分准确的结合自由 能，仅需定性比较结构类似的配体与蛋白质作用能力的 相对强弱. 分子对接方法采用完全经验的打分函数和全 局搜索算法, 可以极其快速的定性判断结合能力强弱. 蒋华良、罗成、郑明月、来鲁华、朱维良、王任小等课 题组在该方向上进行了大量工作 ${ }^{[47-51]}$. 在需要半定量的 场合，采用基于终点(end-point)模拟的方法可以十分便 捷地给出近似的结合能力. 目前最为常用的基于终点模 拟的方法为 MM-G(P)BSA 方法, 侯廷军和张增辉课题 组在近期的综述中对该方法进行了详细的介绍 ${ }^{[52]}$. 另 外，近年提出的 CL-FEP 方法 [53]将中心极限定理与自由 能微扰算法相结合, 使自由能微扰公式适用于终点模 拟，是一种非常有潜力的近似结合自由能计算方法.

\section{5 蛋白质-配体准确结合自由能计算方法的应 用}

蛋白质-配体结合自由能计算已经在药物设计、分 子机器设计、超分子化学等领域得到了广泛的应用. 其 中, Biggin 课题组证明采用基于约束的自由能计算方法, 在力场正确的条件下, 计算结果可以达到化学精度 $(<4.184 \mathrm{~kJ} / \mathrm{mol})^{[54]}$; 罗海涁课题组通过将六自由度约束 和 alchemistry 方法联用, 从候选小分子中篮选出了 15 种可能与新冠病毒主蛋白作用的药物 ${ }^{[55]}$; Jorgensen 课题 组通过计算蛋白质-配体的标准结合自由能, 比较了不 同力场和电荷策略在描述蛋白质-配体化合物中的表 现 ${ }^{[56]}$; Chipot 课题组首次将计算蛋白质-配体准确结合自 由能的思路拓展到蛋白质 - 蛋白质领域, 计算了 barnase-barstar 复合物的准确结合自由能 ${ }^{[57]}$; Rizzi, Mobley 和 Chodera 课题组详尽地讨论了不同策略和软 件实现在解决 “SAMPL6 主客体结合预测挑战” 上的表 现 ${ }^{[58]}$.

本课题组采用基于七自由度约束和重要性采样策 略, 研究了纺锤菌素和 DNA 的结合能力(图 5), 将准确 结合自由能计算方法拓展到了任意主体-客体体系 ${ }^{[59]}$; 探究了碗烯和菲在结合蛋白质能力上的差异, 阐明了具 有立体结构的碗烯在和蛋白质作用上的优势 ${ }^{[60]}$. 由于 诸多研究证明了蛋白质-配体准确结合自由能计算策略 的可靠性, 本课题组将蛋白质-配体标准结合自由能作 为一个考察参数引入力场拟合步骤当中, 并通过计算蛋 白质-配体结合自由能, 验证拟合的力场参数的可靠 性 ${ }^{[61]}$.

鉴于准确性是蛋白质-配体结合自由能计算中最为 重要、核心的问题, 本文总结了部分近年的蛋白质-配体 结合自由能计算与实验值对比工作, 结果进一步验证了 基于约束的蛋白质-配体结合自由能计算的可靠性(表 3). 
表 3 基于约束的蛋白质-配体结合自由能计算与实验值的对比

Table 3 Comparison between calculated and experimental binding free energies

\begin{tabular}{|c|c|c|c|c|}
\hline 作者 & 使用策略 & $\begin{array}{c}\text { 小分子配 } \\
\text { 体个数 }\end{array}$ & $\begin{array}{l}\text { 计算误差/ } \\
\left(\mathrm{kJ} \bullet \mathrm{mol}^{-1}\right)\end{array}$ & 文献 \\
\hline $\begin{array}{l}\text { Aldeghi } \\
\text { 等 }\end{array}$ & alchemistry & 11 & $\mathrm{MAE}=2.5$ & [54] \\
\hline Boyce 等 & alchemistry & 13 & RMSE $=7.5$ & [29] \\
\hline $\mathrm{Li}$ 等 & alchemistr & 100 & RMSE $=2.59 \sim 6.44$ & [30] \\
\hline Laury 等 & alchemistry & 14 & $\mathrm{MAE}=5.02$ & [62] \\
\hline Xie 等 & alchemistry & 141 & $\mathrm{RMSE}=6.65$ & [63] \\
\hline Deng 等 & $\begin{array}{l}\text { alchemistry/ } \\
\text { 重要性采样 }\end{array}$ & 3 & $\begin{array}{l}\mathrm{AE}=6.7 \sim 18(\mathrm{al}- \\
\text { chemistry), } 6.3 \sim \\
14 \text { (重要性采样) }\end{array}$ & [27] \\
\hline $\mathrm{Fu}$ 等 & 重要性采样 & 3(十肽) & $\mathrm{AE}<1.7$ & [23] \\
\hline
\end{tabular}

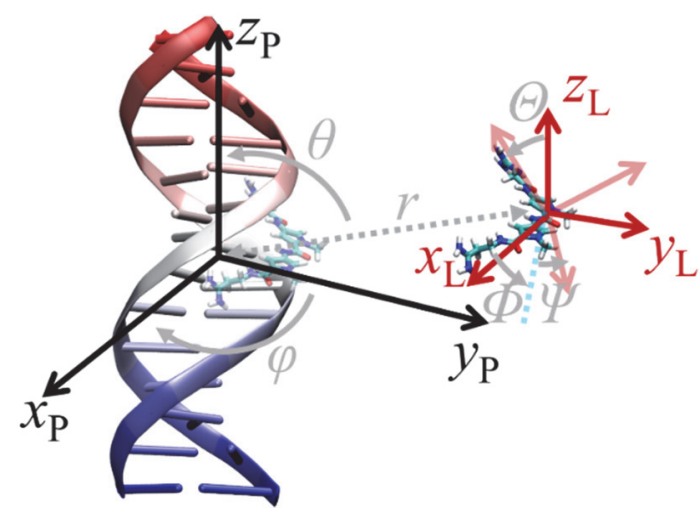

图 5 采用基于最优旋转的七自由度约束结合 WTM-eABF 方法计算 DNA-纺锤菌素的结合自由能

Figure 5 DNA-netropsin binding free-energy calculation by the combination of the optimal-rotation-based seven-degree-of-freedom restraints and the WTM-eABF algorithm

\section{6 总结与展望}

\section{1 总结}

蛋白质-配体问题由于其重要性, 许多年来广受关 注. 本文综述了近年来在采用分子模拟准确计算蛋白 质-配体结合自由能领域的进展, 并对具体的方法和策 略进行了简要的介绍. 在实践中, 一个关键的问题是如 何选择对当前研究的体系最为合适的结合自由能计算 方法, 由于自然界中蛋白质-配体复合物的结构千变万 化, 所以大多数情况下都需要具体问题具体分析, 本文 仅根据本课题组在蛋白质-配体结合自由能计算上的经 验，提出一个基本的建议(表 4).

\section{2 展望}

大量应用实例证明, 对于小分子配体, 现有算法已 经可以准确估计蛋白质-配体标准结合自由能. 然而准 确结合自由能计算仍需较长的时间才能达到收玫, 导致 消耗的计算资源较大, 严重阻碍了该方法在实际中的应 用. 解决该办法的可能途径是开发新型的高效算法加快 采样效率, 降低计算量. 近年来提出的哈密顿副本交换 模拟-自由能微扰(Hamiltonian-replica exchange molecular dynamics-FEP, H-REMD-FEP) ${ }^{[64]}$, 副本交换溶质退
表 4 蛋白质-配体结合自由能计算方法选择建议

Table 4 Suggestion of strategies for protein-ligand binding free-energy estimation

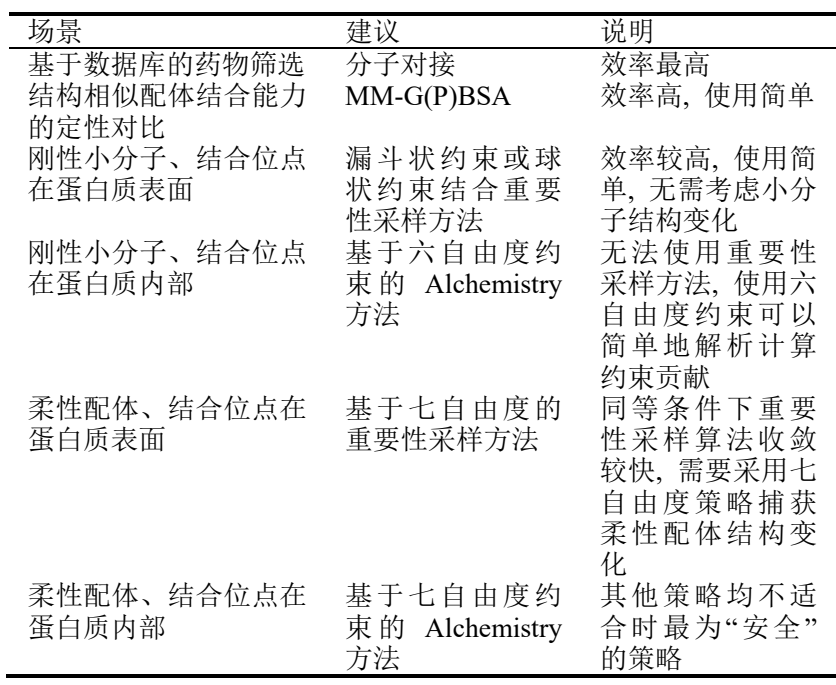

火-自由能微扰(replica exchange with solute scaling, REST2-FEP) ${ }^{[65]}$, 高斯算法加速自由能微扰 (Gaussian algorithm-enhanced FEP, GAFEP) ${ }^{[66]}$, 温度积分采样 metadynamics (integrated tempering samplingmetadynamics, ITS-metadynamics) ${ }^{[67]}$, well-tempered metadynamics-扩展自适应偏置力 (WTM-eABF $)^{[68-69]}$ 等 算法均是在该方向上的尝试, 其中由本课题组提出的 WTM-eABF 方法已经被集成至前述的 BFEE插件中，可 以自动化进行使用.

如何将准确结合自由能计算拓展到更复杂的情况, 如蛋白质-蛋白质结合自由能计算是当前亟需解决的另 一个难题. 由于蛋白质分子较大，对其的“消失”或 “生 长” 不满足 “微扰” 条件, 所以不能使用 alchemistry 方 法进行计算. 另外, 由于蛋白自身的结构远比小分子配 体复杂, 使用约束结合重要性采样方法时需要考虑更多 自由度. Chipot 课题组在计算 barnase-barstar复合物的结 合自由能时，考虑了 14 个自由度，导致计算过程极其复 杂和繁琐 ${ }^{[57]}$. 一种可能的解决途径是提出新型的几何 约束方式, 更加有效地表示蛋白-蛋白间的相对运动模 式 ${ }^{[70]}$. 另一种可能的途径是仅聚焦于对最优自由能路 径的探索, 在得到最优自由能路径之后进一步计算结合 自由能，该策略的一个显著优势是可以得到沿反应路径 的能垒，从而可以同时分析蛋白质-配体的结合效率. 实现该策略的核心是选择一个好的路径采样算法. Roux 课题组利用群轨迹弦方法(strings with swarm of trajectory $)^{[71]}$ 寻找了 barnase-barstar复合物结合-解离的最低自 由能路径, 并利用该路径作为集合变量计算了准确结合 自由能 ${ }^{[22]}$. 但是该方法依旧需要大量计算资源. 通过结 合图论中的路径搜索算法和重要性采样算法 ${ }^{[69]}$, 实现 多维重要性采样计算过程中的实时 (on-the-fly) 路径寻 找 ${ }^{[73]}$ 是高效寻找最优自由能路径的一个可能方法. 


\section{作者简介}

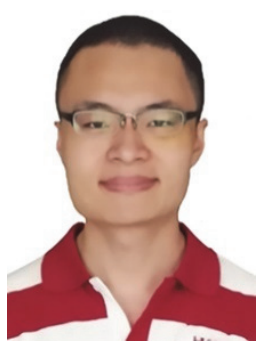

付浩浩, 本科和博士均毕业于南开大学化学系. 现为南 开大学博士后、助理研究员. 研究方向为增强采样算法开发、 蛋白质-配体准确结合自由能计算策略研究和复杂体系中高度 耦合的运动研究.

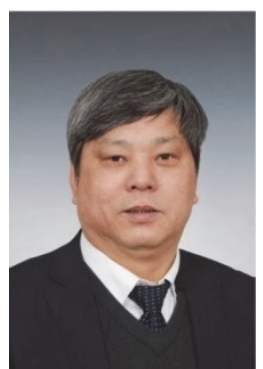

邵学广, 南开大学教授, 博士生导师, 1992 年获中国科学 技术大学中日联合培养博士学位. 2002 年获教育部第三届高 校青年教师奖, 2003 年获国家自然科学基金委杰出青年基金. 主要从事化学计量学及近红外光谱分析方面的研究工作. 建 立了小波变换和免疫算法用于复杂信号解析和在线处理的新 方法以及一系列用于近红外光谱信号处理和建模的化学计量 学方法.

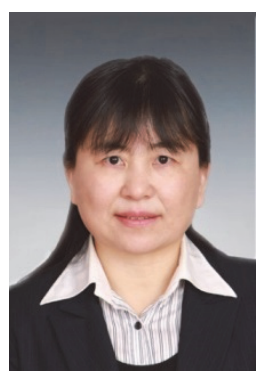

蔡文生, 南开大学教授, 博士生导师. 1994 年获中国科学 技术大学博士学位. 主要从事分子模拟与理论化学计算领域 的研究工作, 包括优化算法、自由能计算方法、分子模拟及理 论化学计算在蛋白质-配体、药物载体、分子机器中的应用研 究.

\section{References}

[1] Chipot, C. Wiley Interdiscip. Rev. Comput. Mol. Sci. 2014, 4, 71.

[2] Siebenmorgen, T.; Zacharias, M. WIREs Comput. Mol. Sci. 2019, 10, e1448.

[3] de Ruiter, A.; Oostenbrink, C. Curr. Opin. Struct. Biol. 2020, 61, 207.

[4] Xu, J.; Wei, Y.; Wu, Z.; Yi, Z. Acta Chim. Sinica 2018, 76, 408 (in Chinese). (徐婕, 魏雨晨, 伍智蔚, 易忠胜, 化学学报, 2018, 76, 408.)

[5] Cournia, Z.; Allen, B. K.; Beuming, T.; Pearlman, D. A.; Radak, B. K.; Sherman, W. J. Chem. Inf. Model. 2020, 60, 4153.
[6] Langan, R. A.; Boyken, S. E.; Ng, A. H.; Samson, J. A.; Dods, G.; Westbrook, A. M.; Nguyen, T. H.; Lajoie, M. J.; Chen, Z.; Berger, S.; Mulligan, V. K.; Dueber, J. E.; Novak, W. R. P.; El-Samad, H.; Baker, D. Nature 2019, 572, 205.

[7] Lemkul, J. A.; Huang, J.; Roux, B.; MacKerell, A. D. Chem. Rev. 2016, 116, 4983.

[8] Fan, Q.; Liang, H.; Xu, X.; Lv, S.; Liang, Z.; Yang, Y. Acta Chim Sinica 2020, 78, 547 (in Chinese). (范勤, 梁洪涛, 许贤祺, 吕松 泰, 梁尊, 杨洋, 化学学报, 2020, 78, 547.)

[9] Nerenberg, P. S.; Head-Gordon, T. Curr. Opin. Struct. Biol. 2018, 49 , 129.

[10] Chipot, C.; Pohorille, A. Free Energy Calculations, Springer, Berlin, Heidelberg, 2007.

[11] Barducci, A.; Bonomi, M.; Parrinello, M. Wiley Interdiscip. Rev Comput. Mol. Sci. 2011, 1, 826.

[12] Laio, A.; Parrinello, M. Proc. Natl. Acad. Sci. 2002, 99, 12562.

[13] Fu, H.; Shao, X.; Cai, W.; Chipot, C. Acc. Chem. Res. 2019, 52, 3254.

[14] Fu, H.; Shao, X.; Chipot, C.; Cai, W. J. Chem. Theory Comput. 2016, 12, 3506 .

[15] Chodera, J. D.; Mobley, D. L. Annu. Rev. Biophys. 2013, 42, 121.

[16] Zwanzig, R. W. J. Chem. Phys. 1954, 22, 1420.

[17] Kirkwood, J. G. J. Chem. Phys. 1935, 3, 300.

[18] Limongelli, V.; Bonomi, M.; Parrinello, M. Proc. Natl. Acad. Sci. 2013, $110,6358$.

[19] Raniolo, S.; Limongelli, V. Nat. Protoc. 2020, 15, 2837.

[20] Capelli, R.; Carloni, P.; Parrinello, M. J. Phys. Chem. Lett. 2019, 10, 3495.

[21] Boresch, S.; Tettinger, F.; Leitgeb, M.; Karplus, M. J. Phys. Chem. B 2003, 107, 9535 .

[22] Woo, H.-J.; Roux, B. Proc. Natl. Acad. Sci. 2005, 102, 6825.

[23] Fu, H.; Cai, W.; Hénin, J.; Roux, B.; Chipot, C. J. Chem. Theory Comput. 2017, 13, 5173.

[24] Torrie, G. M.; Valleau, J. P. J. Comput. Phys. 1977, 23, 187.

[25] Kästner, J. Wiley Interdiscip. Rev. Comput. Mol. Sci. 2011, 1, 932.

[26] Zong, Z.; Li, Q.; Hong, Z.; Fu, H.; Cai, W.; Chipot, C.; Jiang, H.; Zhang, D.; Chen, S.; Shao, X. J. Am. Chem. Soc. 2019, 141, 14451.

[27] Deng, N.; Cui, D.; Zhang, B. W.; Xia, J.; Cruz, J.; Levy, R. Phys. Chem. Chem. Phys. 2018, 20, 17081.

[28] Aldeghi, M.; Heifetz, A.; Bodkin, M. J.; Knapp, S.; Biggin, P. C. J. Am. Chem. Soc. 2017, 139, 946.

[29] Boyce, S. E.; Mobley, D. L.; Rocklin, G. J.; Graves, A. P.; Dill, K. A.; Shoichet, B. K. J. Mol. Biol. 2009, 394, 747.

[30] Li, Z.; Huang, Y.; Wu, Y.; Chen, J.; Wu, D.; Zhan, C. G.; Luo, H. B J. Med. Chem. 2019, 62, 2099.

[31] Tribello, G. A.; Bonomi, M.; Branduardi, D.; Camilloni, C.; Bussi, G. Comput. Phys. Commun. 2014, 185, 604.

[32] Fiorin, G.; Klein, M. L.; Hénin, J. Mol. Phys. 2013, 111, 3345.

[33] Sidky, H.; Colón, Y. J.; Helfferich, J.; Sikora, B. J.; Bezik, C.; Chu, W.; Giberti, F.; Guo, A. Z.; Jiang, X.; Lequieu, J.; Li, J.; Moller, J.; Quevillon, M. J.; Rahimi, M.; Ramezani-Dakhel, H.; Rathee, V. S.; Reid, D. R.; Sevgen, E.; Thapar, V.; Webb, M. A.; Whitmer, J. K.; de Pablo, J. J. J. Chem. Phys. 2018, 148, 44104.

[34] Ibrahim, P.; Clark, T. Curr. Opin. Struct. Biol. 2019, 55, 129.

[35] Wang, J.; Deng, Y.; Roux, B. Biophys. J. 2006, 91, 2798.

[36] de Ruiter, A.; Oostenbrink, C. Curr. Opin. Chem. Biol. 2011, 15, 547.

[37] Lee, T.-S.; Allen, B. K.; Giese, T. J.; Guo, Z.; Li, P.; Lin, C.; McGee, T. D.; Pearlman, D. A.; Radak, B. K.; Tao, Y.; Tsai, H.-C.; Xu, H.; Sherman, W.; York, D. M. J. Chem. Inf. Model. 2020, 60, 5595.

[38] Gumbart, J. C.; Roux, B.; Chipot, C. J. Chem. Theory Comput. 2013, 9, 794.

[39] Karplus, M.; Kuriyan, J. Proc. Natl. Acad. Sci. 2005, 102, 6679.

[40] Rizzi, A.; Grinaway, P. B.; Parton, D. L.; Shirts, M. R.; Wang, K.; Eastman, P.; Friedrichs, M.; Pande, V. S.; Branson, K.; Mobley, D. L.; Chodera, J. D. "YANK: A GPU-accelerated platform for alchemical free energy calculations," can be found under getyank.org, 2020.

[41] Mobley, D. L.; Chodera, J. D.; Dill, K. A. J. Chem. Theory Comput. 2007, 3, 1231.

[42] Henriksen, N. M.; Fenley, A. T.; Gilson, M. K. J. Chem. Theory Comput. 2015, 11, 4377.

[43] Heinzelmann, G.; Henriksen, N. M.; Gilson, M. K. J. Chem. Theory Comput. 2017, 13, 3260.

[44] Cruz, J.; Wickstrom, L.; Yang, D.; Gallicchio, E.; Deng, N. J. Chem. Theory Comput. 2020, 16, 2803. 
[45] Coutsias, E. A.; Seok, C.; Dill, K. A. J. Comput. Chem. 2004, 25, 1849.

[46] Fu, H.; Gumbart, J. C.; Chen, H.; Shao, X.; Cai, W.; Chipot, C. J. Chem. Inf. Model. 2018, 58, 556.

[47] Xing, J.; Lu, W.; Liu, R.; Wang, Y.; Xie, Y.; Zhang, H.; Shi, Z.; Jiang, H.; Liu, Y. C.; Chen, K.; Jiang, H.; Luo, C.; Zheng, M. J. Chem. Inf. Model. 2017, 57, 1677.

[48] Wang, Y.; Li, L.; Zhang, B.; Xing, J.; Chen, S.; Wan, W.; Song, Y.; Jiang, H.; Jiang, H.; Luo, C.; Zheng, M. J. Med. Chem. 2017, 60, 2026.

[49] Zhang, X.; Li, X.; Wang, R. J. Chem. Inf. Model. 2009, 49, 1033.

[50] Pei, J.; Wang, Q.; Liu, Z.; Li, Q.; Yang, K.; Lai, L. Proteins Struct. Funct. Bioinforma. 2006, 62, 934.

[51] Liu, Y.; Xu, Z.; Yang, Z.; Chen, K.; Zhu, W. J. Mol. Model. 2013, $19,5015$.

[52] Wang, E.; Sun, H.; Wang, J.; Wang, Z.; Liu, H.; Zhang, J. Z. H.; Hou, T. Chem. Rev. 2019, 119, 9478.

[53] Ruiz-Blanco, Y. B.; Sanchez-Garcia, E. J. Chem. Theory Comput. 2020, 16, 1396.

[54] Aldeghi, M.; Heifetz, A.; Bodkin, M. J.; Knapp, S.; Biggin, P. C. Chem. Sci. 2016, 7, 207.

[55] Li, Z.; Li, X.; Huang, Y.-Y.; Wu, Y.; Liu, R.; Zhou, L.; Lin, Y.; Wu, D.; Zhang, L.; Liu, H.; Xu, X.; Yu, K.; Zhang, Y.; Cui, J.; Zhan, C. G.; Wang, X.; Luo, H. B. Proc. Natl. Acad. Sci. 2020, 117, 27381.

[56] Qian, Y.; Cabeza de Vaca, I.; Vilseck, J. Z.; Cole, D. J.; Tirado-Rives, J.; Jorgensen, W. L. J. Phys. Chem. B 2019, 123, 8675.

[57] Gumbart, J. C.; Roux, B.; Chipot, C. J. Chem. Theory Comput. 2013, 9, 3789 .

[58] Rizzi, A.; Jensen, T.; Slochower, D. R.; Aldeghi, M.; Gapsys, V.; Ntekoumes, D.; Bosisio, S.; Papadourakis, M.; Henriksen, N. M.; de
Groot, B. L.; Cournia, Z.; Dickson, A.; Michel, J.; Gilson, M. K.; Shirts, M. R.; Mobley, D. L.; Chodera, J. D. J. Comput. Aided. Mol. Des. 2020, 34, 601 .

[59] Zhang, H.; Gattuso, H.; Dumont, E.; Cai, W.; Monari, A.; Chipot, C.; Dehez, F. Molecules 2018, 23, 228.

[60] Du, S.; Wang, H.; Yang, Y.; Feng, X.; Shao, X.; Chipot, C.; Cai, W. J. Phys. Chem. C 2019, 123, 922.

[61] Liu, H.; Fu, H.; Shao, X.; Cai, W.; Chipot, C. J. Chem. Theory Comput. 2020, 16, 6397.

[62] Laury, M. L.; Wang, Z.; Gordon, A. S.; Ponder, J. W. J. Comput. Aided. Mol. Des. 2018, 32, 1087.

[63] Xie, B.; Nguyen, T. H.; Minh, D. D. L. J. Chem. Theory Comput. 2017, 13, 2930 .

[64] Jiang, W.; Roux, B. J. Chem. Theory Comput. 2010, 6, 2559.

[65] Jo, S.; Jiang, W. Comput. Phys. Commun. 2015, 197, 304.

[66] Miao, Y.; Feher, V. A.; McCammon, J. A. J. Chem. Theory Comput. 2015, 11, 3584.

[67] Yang, Y. I.; Niu, H.; Parrinello, M. J. Phys. Chem. Lett. 2018, 9, 6426.

[68] Fu, H.; Zhang, H.; Chen, H.; Shao, X.; Chipot, C.; Cai, W. J. Phys. Chem. Lett. 2018, 9, 4738.

[69] Fu, H.; Chen, H.; Wang, X.; Chai, H.; Shao, X.; Cai, W.; Chipot, C. J. Chem. Inf. Model. 2020, 60, 5366 .

[70] Perthold, J. W.; Oostenbrink, C. J. Chem. Theory Comput. 2017, 13, 5697.

[71] Pan, A. C.; Sezer, D.; Roux, B. J. Phys. Chem. B 2008, 112, 3432.

[72] Suh, D.; Jo, S.; Jiang, W.; Chipot, C.; Roux, B. J. Chem. Theory Comput. 2019, 15, 5829.

[73] Brotzakis, Z. F.; Limongelli, V.; Parrinello, M. J. Chem. Theory Comput. 2019, 15, 743.

(Cheng, B.) 